\title{
CENTRAL RETINAL ARTERY AND CENTRAL OPTIC NERVE ARTERY*
}

\author{
BY
}

\author{
J. FRANÇOIS AND A. NEETENS
}

From the Ophthalmological Clinic, University of Ghent, Belgium (Director: Prof. J. François).

SiNCE our last publications (François and Neetens, 1954, 1956; François, Neetens, and Collette, 1955; François, Verriest and Baron, 1956; François, Verriest, Neetens, and De Rouck, in the press) on the blood supply to the optic nerve other studies on this subject have appeared in the literature. These relate to three groups of questions.

\section{(I) Does a central optic nerve artery indeed exist?}

As we shall demonstrate below, on the basis of more recent observations, the existence of such an artery in the optic nerve is beyond all doubt. Besides the anatomical observations we have clinical proof of its presence; there is a syndrome of acute loss of vision similar to that due to obstruction of the central retinal artery, which involves the optic nerve (vascular pseudopapillitis).

Whereas Singh and Dass (1960a, b) neither deny nor mention its existence, Dejean, Viallefont, Boudet, and Costeau (1957) definitely deny the existence of such an artery; Steele and Blunt (1956) mention the presence of an artery lying in the centre of the optic nerve and originating from the central retinal artery. Renard (1961) denies the existence of such an artery, although his figures actually favour its existence. Lazorthes, Daraux, and Gaubert (1961) have also demonstrated the existence of this artery.

(II) Do branches of the central retinal artery exist?

(1) Does the central retinal artery give off branches before it reaches the optic nerve or its sheath?

Steele and Blunt (1956) and Singh and Dass (1960a, b) think it does, whereas Dejean and others (1957) think it does not. In our opinion the central retinal artery sometimes gives off a few branches in this part of its course. Sometimes, however, there is but one branch or even no branch at all, from which we may conclude that branching is rare and variable.

(2) Does the central retinal artery give off branches at the sheath of the optic nerve itself?

Again, Steele and Blunt (1956) and Singh and Dass (1960) think it does but Dejean and others (1957) do not. It is a general rule that all arteries approaching the sheath or neurilemma of a nerve supply the latter with branches

\footnotetext{
* Received for publication April 24, 1962.
} 
shortly before or on reaching the sheath. Each artery has only a few branches, but many arteries participate in the vascular supply of the sheath. This general rule also applies to the central retinal artery; at the site of the sheath it gives off one branch (or very rarely two) in a constant fashion. The diameter of these branches is relatively small.

\section{(3) Does the central retinal artery give off intraneural branches?}

Steele and Blunt (1956) and Singh and Dass (1960) claim that the artery has many such branches, whereas Dejean and others (1957) saw none. According to Bignell (1952), a few branches might sometimes be given off after the lamina cribrosa, which he thought to supply the optic nerve.

Immediately after the lamina cribrosa and sometimes slightly further back, but always in the anterior third of the intraneural course of the central retinal artery, the latter may give off a few branches.

Detailed examination of the data published by Singh and Dass (1960) regarding intraneural branches of the central retinal artery, yielded the following percentages: in only 17.2 per cent. of cases the central retinal artery has four or more branches (four branches 10.9 per cent.; five branches 0 per cent.; six branches 1.6 per cent.; seven branches 0 per cent.; eight branches 1.6 per cent.; dubious 3.1 per cent.) In 82.8 per cent. of cases the central retinal artery has $0-3$ branches $(0$ branches 25 per cent.; one branch $26 \cdot 6$ per cent.; two branches 20.3 per cent.; three branches 10 per cent). Singh and Dass (1960) do not mention the exact site where most of these branches are given off. Thus it is seen that, in the majority of cases ( 82.8 per cent.), the central retinal artery has one or two intraneural branches or none. These figures agree well with our own findings.

(4) Does the central retinal artery give off branches at the level of the lamina cribrosa?

Dejean and others (1957) state that there are numerous branches at this site. Steele and Blunt (1956), Singh and Dass (1960), and the present authors observed none.

(III) Does the central retinal artery form anastomoses with other vascular regions, either by itself or by its branches?

Only Dejean and others (1957) mention broad anastomoses between the central retinal artery and the vessels of the Zinn-Haller circle, the lamina cribrosa. According to our observations there are no anastomoses at any level, including the lamina cribrosa. According to Singh and Dass (1960), however, recurrent branches of the Zinn-Haller circle form anastomoses in the pia mater with the pial branches of the central retinal artery. Further on the problematical nature of this question will be discussed. 
From this review it becomes clear that, besides these fundamental differences of opinion, there are other differences that may be brought into agreement by a discussion of the methods used. As regards the true differences of opinion, new proofs will be given in the present article.

\section{Observations}

What is the origin of the central retinal artery and the central optic nerve artery, how do they give off branches, and what relationship exists between them?

Careful dissection of the branches of the ophthalmic artery in 31 orbits has led us to formulate the diagrams shown in Fig 1.
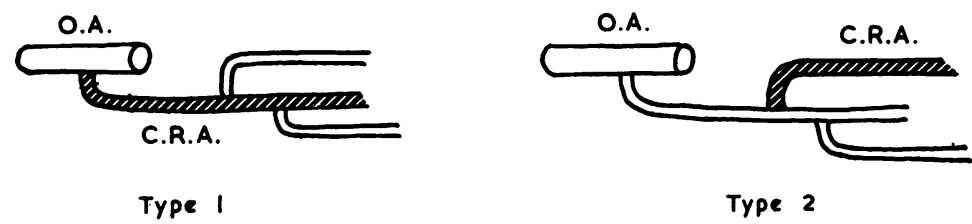

Type 2

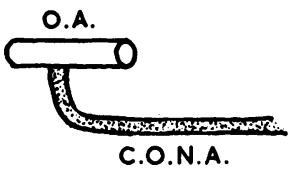

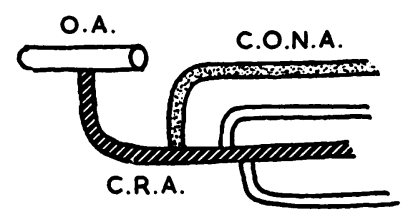

Type b

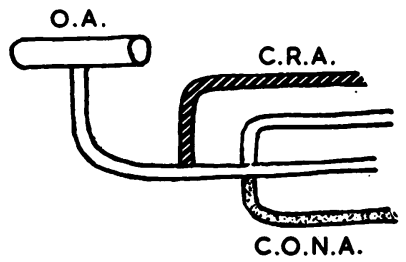

Type c

FIG. 1.-Schematic representation of different origins of central retinal artery (C.R.A.) and central optic nerve artery (C.O.N.A.) in relation to ophthalmic artery (O.A.).

In most cases the central retinal artery branches off immediately from the ophthalmic artery (Type 1, 26 cases). It may also derive from a common branch, which also gives origin to dural, muscular, and ciliary arteries (Type 2, 5 cases).

The central optic nerve artery may originate immediately from the ophthalmic artery (Type a, 7 cases) or be a branch of the central retinal artery (Type b, 2 cases, one intraneural and one extraneural).

There is also the possibility that both the central retinal artery ( 5 cases) and the central optic nerve artery (1 case) originate from a common stem of the ophthalmic artery, from which other arterioles also derive (Type c). In no case was the central retinal artery dependent on the central optic nerve artery; the diameter of the latter is invariably smaller than that of the former, 0.2 or $0.1 \mathrm{~mm}$. (4 cases), as against $0.25 \mathrm{~mm}$. 


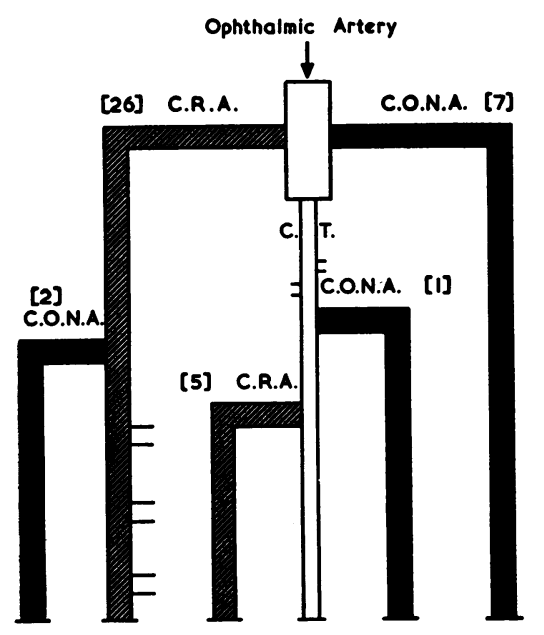

FIG. 2.-Origin of central retinal artery (C.R.A.) and central optic nerve artery (C.O.N.A.) in our 31 specimens.

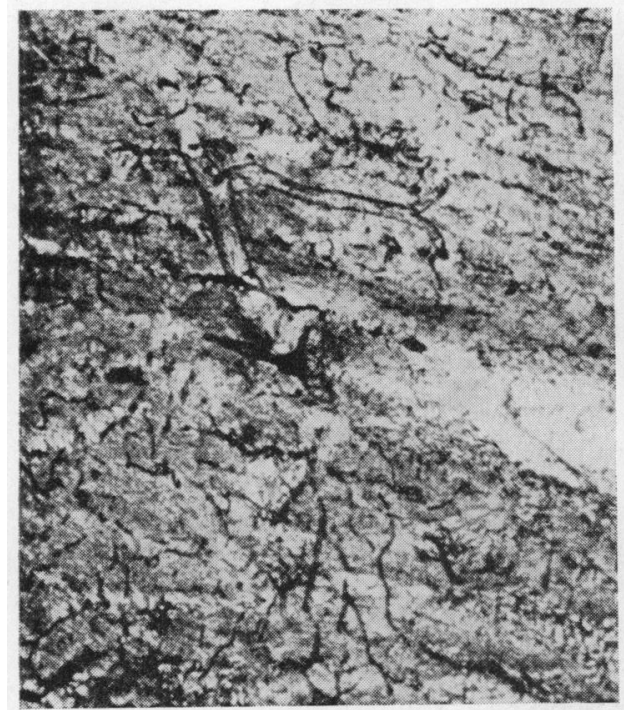

FIG. 3.-Longitudinal section of human optic nerve. Middle part, showing central optic nerve artery on the left and central retinal vessels on the right. Benzidine stain. $\times 16$.
The central retinal artery was present in all 31 cases, and the central optic nerve artery in ten of them (Fig. 2). Thus at the level of the optic nerve, an axial vascular system is present in only about one third of cases (Figs 3 and 4). In some cases the central retinal artery gave off a small axial branch towards the optic nerve; in two cases there was a small satellite arteriole of the central retinal artery which branched off at the level of the ophthalmic artery, penetrated the optic nerve at a site some $15 \mathrm{~mm}$. behind the globe, and then gave rise to an anterior and posterior branch inside the optic nerve. It is indeed surprising that Singh and Dass (1960) never found any such

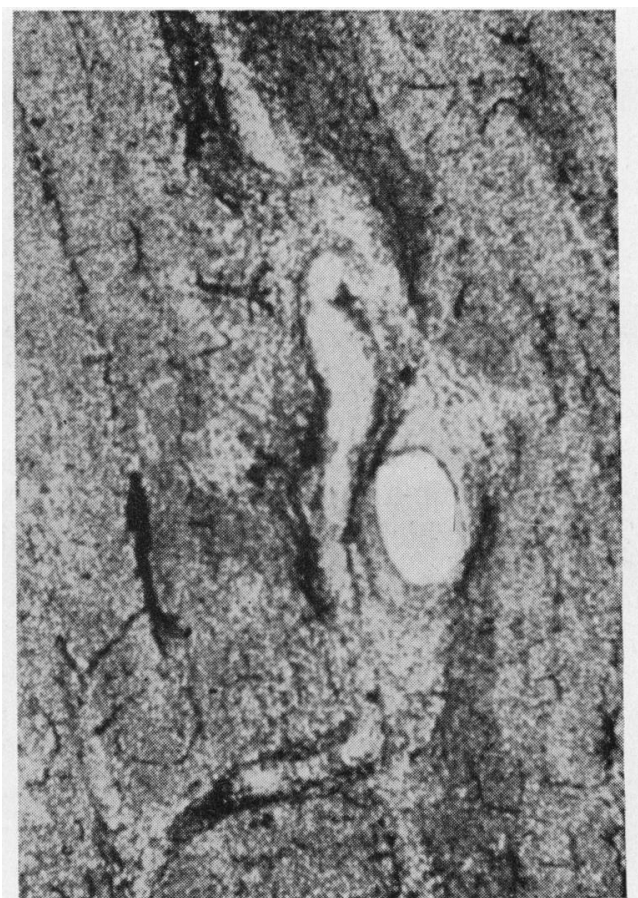

FIG. 4.- - Longitudinal section of human optic nerve. Middle part, showing central optic nerve artery on the left and central retinal vessels on the right. Benzidine stain. $\times 25$. 
branch. In three cases the central optic nerve artery gave off only one posterior intraneural branch. Consequently this axial supplying system can only occur in the posterior part of the nerve.

Besides anatomical proof there is also clinical proof of the existence of the central optic nerve artery. In a case of acute loss of vision, in which the classic fundus picture of the central retinal artery embolism is absent, besides acute papillitis or acute retrobulbar neuritis, one should consider embolism of an important optic nerve artery (pseudo-papillitis), especially if there is also a general vascular disorder (endarteritis obliterans, atherosclerosis).

\section{Does the central retinal artery give off branches?}

As we have already pointed out, the central retinal artery gives off branches outside the sheath of the optic nerve; these branches supply the sheath of the optic nerve, and may then participate in the supply of the periphery of the nerve, though this is somewhat rare (eight of the 31 cases). Moreover, at the site where the central retinal artery penetrates the nerve (together with the central optic nerve artery if this exists), some $10-15 \mathrm{~mm}$. behind the globe, a great and variable number of arterioles of other origin may also penetrate the nerve. Therefore we may conclude that in the first case at the level of the sheath, and outside it, the central retinal artery gives off one and at most two branches in a constant fashion, before reaching the optic nerve. From a nutritional point of view these branches are not very important, since numerous arterioles originating from other arteries also supply the sheath. In the second case, the central retinal artery may give off branches in an irregular fashion, before it reaches the sheath. The number of these branches is very small, and there may be none at all.

As we stated in our earlier studies on the vascularization of the optic nerve, we repeatedly observed one or two intraneural branches. These usually originated from the central retinal artery in the anterior third part of its intraneural course, immediately behind the lamina cribrosa. They ran perpendicularly to the central retinal artery, turned rapidly towards the front, and running parallel to the central retinal artery reached the disc. Here they divided into numerous branches and penetrated the retinal vascular network; these were clearly early retinal branches. They are always of an arteriolar or pre-capillary nature and have no nutritional function until they reach the capillary stage in the peri-papillary part of the retina.

At the level of the lamina cribrosa the central retinal artery was never observed to give off a branch.

The disagreement regarding the branches of the central retinal artery is only apparent. We may draw the conclusion that the central retinal artery has branches, though these are rare. From a physiological point of view, obstruction of these branches will not interfere with the nourishment of the nerve. In each vascular network there are variants at the arteriolar stage, but in the final capillary stage the distribution is constant. 
It seemed desirable to experiment with injections and determine the filling of the tissues dependent on the artery injected on a physiological-anatomical basis. One very important precaution has been neglected so far: viz. thorough previous elimination of the venous system. This is one reason why Bignell (1952) reached erroneous conclusions. The injected medium returns via the capillary network through the veins. Since the latter possess many anastomoses, the regions where filling is observed are erroneously thought to possess arterial distribution. Our micro-radiographic method also makes it difficult to distinguish between arterial and venous distribution. We have overcome this difficulty by the following technique: a medium with very large particles (15-25 $\mu$ and more) is injected into the central retinal artery under low pressure and very slowly to avoid rupture of the vascular wall and consequent false results; by this means no return via the venous system is possible, since the medium cannot pass through the capillary network. On injection of a similar medium, but with smaller particles $(2-6 \mu)$ the greater portion will pass through the capillary network and a distinct return via the venous system is observed.

In this way we obtained a first image (Fig. 5). In the middle there is a

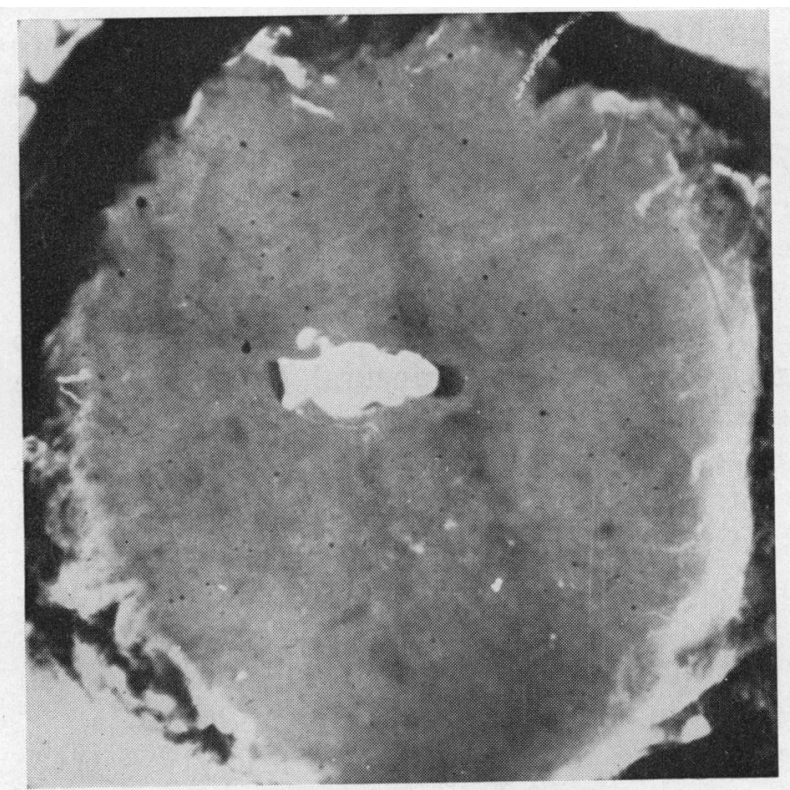
distinct central retinal artery, there is no venous filling, the nerve is empty, except for the periphery, where a few pial branches allow a very small degree of filling. A branch of the central retinal artery that immediately turns forwards may also be seen.

FIg. 5.-Transverse section of human optic nerve. Anterior part. Chlorbismol injection. $\times 14$.

Fig. 6 (opposite) shows the results after injecting a medium with smaller particles: irregular filling of the optic nerve, dependent on the venous branches. The central retinal artery is seen and this again has an early retinal branch.

These illustrations all represent the region immediately behind the lamina cribrosa, or slightly further backwards. 

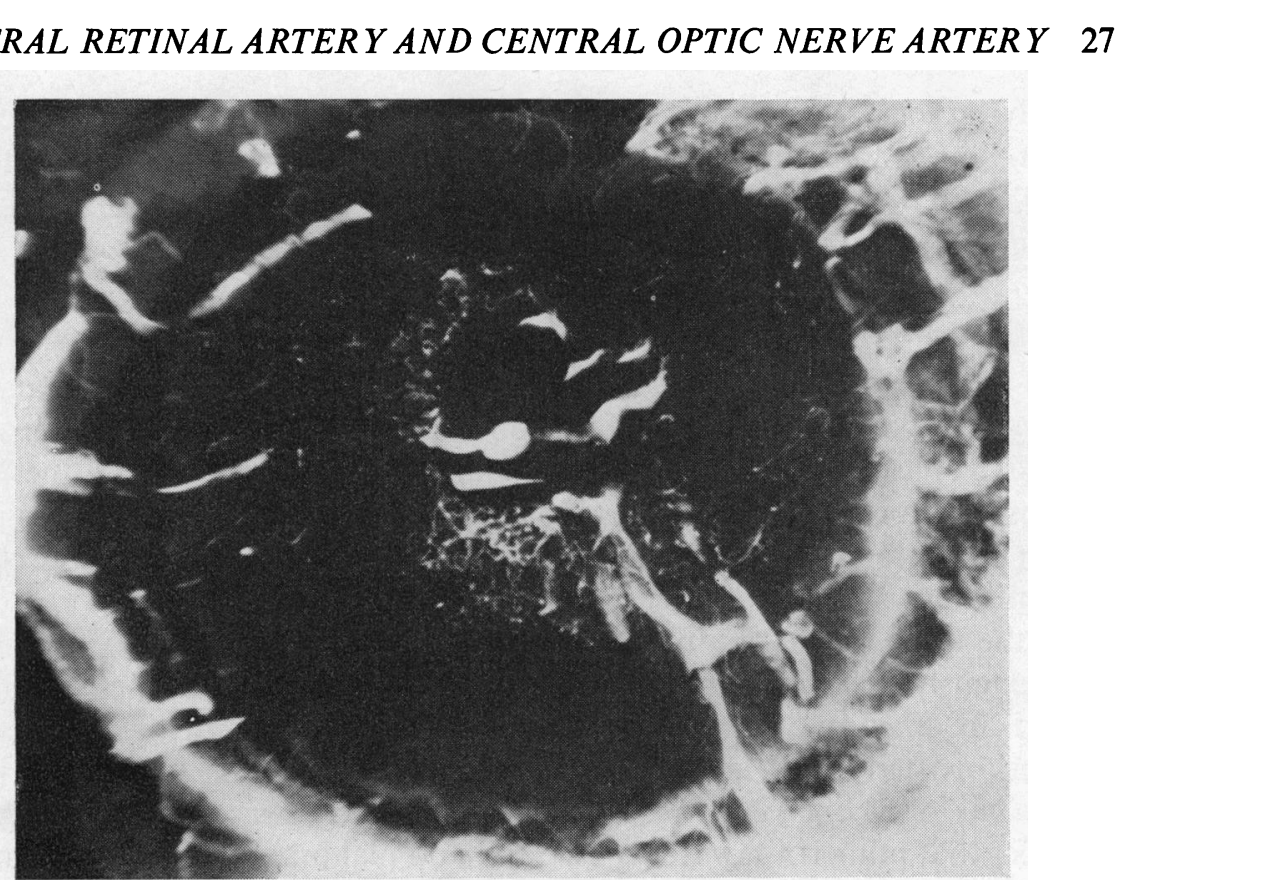

Fig. 6. -Transverse section of human optic nerve. Anterior part. Angiopac injection. $\times 14$.

A final experiment demonstrates that the nutrition of the optic nerve does not depend on the central retinal artery. Instead of injecting the central retinal artery we eliminated it by ligating its origin. The ophthalmic artery was then injected; Fig. 7 shows the anterior third part of the intra-orbital optic nerve. In the middle are some blood-vessels of pre-capillary order or

Fig. 7.-Transverse section of human optic nerve. Anterior part. Thorotrast injection. $\times 14$.

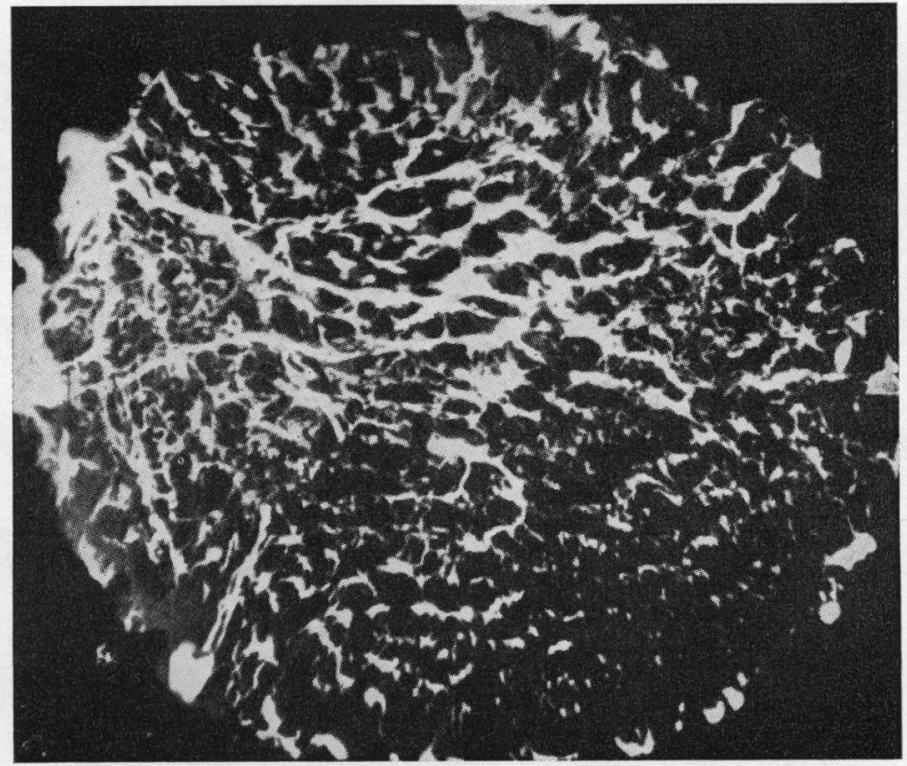


slightly larger. The central retinal artery is not shown, but the optic nerve is completely and excellently filled, as is the network at the level of the pia mater, which contains several large blood vessels, more than in the first experiment.

\section{Does the central retinal artery anastomose with other vascular regions?}

We were unable to demonstrate any anastomoses at any level whatsoever. Dejean and others (1957) are the only authors who have demonstrated extensive anastomoses with the Zinn-Haller circle at the level of the lamina cribrosa. However, their illustrations are far from convincing. Singh and Dass (1960) think that recurrent pial branches of the Zinn-Haller circle may communicate with pial branches of the central retinal artery.

At the level of the pia mater and the sheath of the optic nerve there is a very extensive arterial network. It was our aim to decide whether this was indeed an anastomotic network and Fig 8 clearly demonstrates that such a network existed at all levels. We have also proved that the central retinal artery has only one or two branches at the optic nerve sheath. If we remember that numerous muscular, ciliary, and pial branches of the ophthalmic artery supply the pial network, it seems impossible that recurrent pial branches of the Zinn-Haller circle may form anastomoses with the small number of pial branches of the central retinal artery. Our studies confirm that the Zinn-Haller circle communicates with the extensive pial network, but we were not able to prove that a pial branch of the central retinal artery was especially involved in these anastomoses.

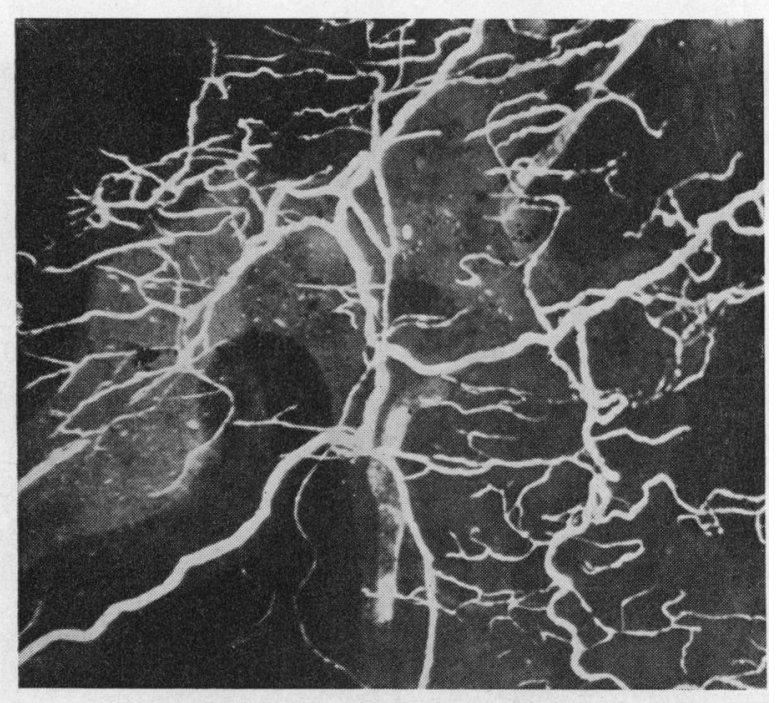

Fig. 8.-Human optic nerve. Pial sheath. Thorotrast injection, showing rich anastomotic arterial network. $\times 24$.

The central retinal artery never forms anastomoses with other vascular regions and therefore constitutes a true final artery. 


\section{Conclusions and Summary}

The blood supply of the optic nerve by the central retinal artery and the central optic nerve artery is summarized in the Table.

TABLE

BLOOD SUPPLY OF CENTRAL RETINAL AND CENTRAL OPTIC NERVE ARTERIES

\begin{tabular}{l|l|l}
\hline Central Optic Nerve Artery. & \multicolumn{2}{|c}{ Present in one third of cases } \\
\hline & $\begin{array}{l}\text { Extraneural } \\
\text { Extrapial }\end{array}$ & Inconstantly 1-2 branches \\
\cline { 2 - 3 } Central Retinal Artery & Pial & Constantly 1-2 branches \\
\cline { 2 - 3 } & Intraneural & $\begin{array}{l}\text { Inconstantly in its anterior } \\
\text { third early retinal branches }\end{array}$ \\
\cline { 2 - 3 } & Lamina cribrosa & No branches \\
\hline & Anastomoses & None \\
\hline
\end{tabular}

Clinically and anatomically there exists a central optic nerve artery (in ten of our 31 cases). There also exists a syndrome of occlusion of this central optic nerve artery (vascular pseudo-papillitis). The differential diagnosis of this disorder should include occlusion of the central retinal artery and acute papillitis.

The central retinal artery and the central optic nerve artery may originate from the ophthalmic artery in various ways. The central retinal artery can give off small branches-in an irregular fashion-before reaching the sheath of the optic nerve. These branches finally reach the vascular network of the pia mater. At the optic nerve sheath the central retinal artery regularly gives off one or at most two branches, which are incorporated into the anastomotic arterial network of the pia mater.

In its intraneural course the central retinal artery may give off a few branches in the anterior third of its course behind the lamina cribrosa. At the lamina cribrosa the central retinal artery never gives off branches. There are no intraneural anastomoses between the central retinal artery and other vascular regions. The Zinn-Haller circle anastomoses with the very extensive arterial network of the pia mater. It cannot be determined whether branches of the central retinal artery are especially involved in these anastomoses.

\section{REFERENCES}

Bignell, J. L. (1952). Trans. ophthal. Soc. Aust., 12, 105.

Dejean, C., Viallefont, Boudet, and Costeau (1957). Bull. Soc. Ophtal. Fr., p. 548.

François, J., and NeEtens, A. (1954). Brit. J. Ophthal., 38, 472.

(1956). Ibid., 40, 45 . 
François J., Neetens, A., and Collette, J. M. (1955). I Ibid., 39, 220. VERRIEST, G., and BARON, A. (1956). Bull. Soc. franc. Ophtal., 69, 36.

,-- , NeETEns, A., and De RoucK, A. Ann. Oculist. (Paris), (in press).

LazorThes, G., DarauX, H., and GAUBert, J. (1961). Bull. Soc. franc. Ophtal., 74, 276.

RENARD, G. (1961). Ibid., 74, 290.

SingH, S., and DaSS, R. (1960a). Brit. J. Ophthal., 44, 193.

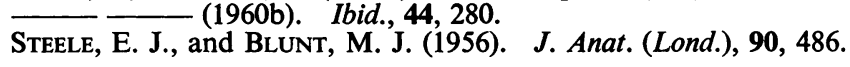

